\title{
Efficiency First or Equity First? - Two Principles and Rationality of Social Choice
}

\author{
Koichi Tadenuma* \\ October 1996 \\ Revised version: June 1998
}

\begin{abstract}
The Pareto efficiency criterion is often in conflict with the equity criteria as no-envy or as egalitarian-equivalence: An allocation $x$ that is Pareto superior to another allocation $y$ can be inferior to $y$ in consideration of equity. This paper formalizes two different principles of social choice under possible conflict of efficiency and equity. The efficiency-first principle requires that we should always select from efficient allocations, and when the efficiency criterion is not at all effective as a guide for selection, i.e., when all the available allocations are efficient or there is no efficient allocation, we should apply an equity criterion to choose desirable allocations. The equity-first principle reverses the lexicographic order of application of the two criteria. We examine rationality of the social choice rules satisfying these two principles. It is shown that the degree of rationality varies widely depending on which principle the social choice rules represent. Several impossibility and possibility results as well as a characterization theorem are obtained.
\end{abstract}

JEL Classification Numbers: D71, D63, D61.

*Department of Economics, Hitotsubashi University, Kunitachi, Tokyo 186-8601, Japan. Fax: (81)-42-580-8748. E-Mail: tadenuma@econ.hit-u.ac.jp 


\section{Introduction}

Most economists accept the Pareto efficiency criterion as a criterion of social desirability of resource allocations: An allocation $x$ is Pareto superior to an allocation $y$ if no one is worse off and someone is strictly better off at $x$ than at $y$. An allocation is Pareto efficient in a given set of available allocations if no available allocation is Pareto superior to it. However, the Pareto criterion is silent about the distributional equity of allocations but concerns only efficient use of resources.

On the other hand, several concepts of distributional equity have been extensively studied recently in economics. Two of them are central: no-envy and egalitarian-equivalence. ${ }^{1}$ An allocation is envy-free if no one prefers the consumption bundle of any other agent to his own. An allocation $x$ meets egalitarian-equivalence if and only if there is a consumption bundle $x_{0}^{*}$ such that every agent is indifferent between his bundle at $x$ and the bundle $x_{0}^{*}$. Particular subclasses of egalitarian equivalent allocations are also considered by fixing the direction of the reference consumption vectors. Given a vector $\bar{r}$, an allocation $x$ satisfies $\bar{r}$-egalitarian-equivalence if and only if there is a real number $t \in \mathbf{R}$ such that every agent is indifferent between his bundle at $x$ and the bundle $t r$. Each of these equity concepts does not depend on cardinal utilities of agents, but is based only on ordinal preferences just as the Pareto criterion is.

However, as Feldman and Kirman (1974) pointed out, there is a fundamental conflict between the Pareto efficiency criterion and the equity-asno-envy criterion. There often exist two allocations $x$ and $y$ such that $x$ is Pareto superior to $y$ whereas $x$ is not envy-free but $y$ is. The two criteria are completely opposed to each other on which allocation is socially desirable. Hence, if these two allocations $\{x, y\}$ are the only policy options available at the time, we cannot attain an allocation that is both Pareto efficient in $\{x, y\}$ and envy-free, but we have to choose either an efficient allocation or an envy-free allocation. The same kind of conflict also arises between the Pareto efficiency criterion and the equity-as-egalitarian-equivalence criterion as shown in the present paper.

When two criteria of decision-makings are mutually inconsistent, it is natural to take one criterion as the first principle and the other as the sec-

\footnotetext{
${ }^{1}$ The concept of no-envy was introduced by Tinbergen(1953), Foley(1967) and Kolm(1972), and that of egalitarian-equivalence by Pazner and Schmeidler(1978).
} 
ond. Some might think, as most economists do, that efficiency should be considered first, while others might argue that distributional equity should always be kept for social stability. This paper formalizes these ideas. In the efficiency-first principle, we require that choice should always be made from efficient allocations whenever there are any. Then, an equity criterion should be applied, as the second principle, when the efficiency criterion is not at all effective as a guide for selection, namely either when all the allocations available are efficient or when there is no efficient allocation at all. In the equity-first principle, we reverse the order of application of the two criteria.

Requiring social choice correspondences to satisfy the efficiency-first and equity-second principle or the equity-first and efficiency-second principle, we study a classical issue in social choice theory: rationality of choice correspondences. Several degrees of rationality properties are considered. To examine these properties is especially important as they are logically related to path independence of social choice: "the independence of the final choice from the path to it" (Arrow, 1963, p. 120). Path independence is an indispensable property of social choice rules. Were it violated, some arbitrary agenda controls could affect the final choice, which is clearly undesirable.

We obtain several impossibility theorems on the existence of social choice correspondences satisfying the efficiency-first and equity-second-as-no-envy principle (or equity-second-as-egalitarian-equivalence), and the weakest requirement of rationality known as Chernoff's axiom (which is a necessary condition for path independence). With $\bar{r}$-egalitarian-equivalence as the equity concept, there exists a social choice correspondence satisfying the above properties. However, if we strengthens the rationality requirement to path independence, we reach another impossibility.

By contrast, we establish the existence of social choice correspondences satisfying the equity-first and efficiency-second principle, and quasi-transitive rationality (which is a sufficient condition for path independence). This possibility result holds with any concept of equity considered in this paper. Moreover, we completely identify the social choice correspondence that satisfies the equity-first and efficiency-second principle, path independence, and some additional properties.

It is often argued whether we should consider efficiency first or equity first when we evaluate social desirability of allocations. Our results show that possibility of consistent social decisions (in the sense of path independence) depends crucially on which philosophical position we take. 


\section{Preliminaries}

There are $n$ agents and $m$ infinitely divisible goods, where $n$ and $m$ are some finite numbers. Let $N=\{1, \cdots, n\}$ be the set of agents. Denoting by $\mathbf{R}$ the set of real numbers, the set $\mathbf{R}_{+}^{m}$ is the consumption set of each agent. Let $\mathcal{R}$ be the class of preference relations on $\mathbf{R}_{+}^{m}$ that are reflexive, transitive, complete, continuous, and monotonic. Each agent $i \in N$ is endowed with a preference relation $R_{i}$ in $\mathcal{R}$. The strict preference relation and the indifference relation of agent $i$ are denoted by $P_{i}$ and $I_{i}$, respectively. A list of preference relations, $\left(R_{i}\right)_{i \in N} \in \mathcal{R}^{n}$, is called a preference profile, and denoted by $R_{N}$.

An allocation is a vector $x=\left(x_{1}, \cdots, x_{n}\right) \in \mathbf{R}_{+}^{m n}$ where each $x_{i}=$ $\left(x_{i 1}, \cdots, x_{i m}\right) \in \mathbf{R}_{+}^{m}$ is a consumption bundle of agent $i \in N$. The set of all allocations is denoted by $X{ }^{2}$ Let $\mathcal{S}$ be the set of all finite subsets of $X$.

Let a preference profile $R_{N} \in \mathcal{R}^{n}$ be given. An allocation $x \in X$ is Pareto superior to an allocation $y \in X$ at $R_{N}$ if $x_{i} R_{i} y_{i}$ for all $i \in N$ and $x_{i} P_{i} y_{i}$ for some $i \in N$. If $x \in X$ is Pareto superior to $y \in X$ at $R_{N}$, we write $x \succ_{P\left(R_{N}\right)} y$. For each $S \in \mathcal{S}$, an allocation $x \in S$ is Pareto efficient in $S$ at $R_{N}$ if there is no allocation $y \in S$ such that $y \succ_{P\left(R_{N}\right)} x$. Let $P\left(R_{N}, S\right)$ be the set of Pareto efficient allocations in $S$ at $R_{N}$. An allocation $x \in X$ is envyfree at $R_{N}$ if $x_{i} R_{i} x_{j}$ for all $i, j \in N$. For each $S \in \mathcal{S}$, let $F\left(R_{N}, S\right)$ be the set of envy-free allocations in $S$ at $R_{N}$. An allocation $x \in X$ is egalitarianequivalent at $R_{N}$ if there is a consumption bundle $x_{0} \in \mathbf{R}_{+}^{m}$ such that for all $i \in N, x_{i} I_{i} x_{0}$. Then, the bundle $x_{0}$ is called a reference bundle for $x$. For each $S \in \mathcal{S}$, let $E\left(R_{N}, S\right)$ be the set of egalitarian-equivalent allocations in $S$ at $R_{N}$. Particular subclasses of egalitarian-equivalent allocations are often studied in the literature. Let $\bar{r} \in \mathbf{R}_{++}^{m}$ be a given vector. An allocation $x \in X$ is egalitarian-equivalent for a fixed reference ray with $\bar{r}$ at $R_{N}$ or simply $\bar{r}$-egalitarian-equivalent at $R_{N}$ if there is a real number $t \in \mathbf{R}$ such that for all $i \in N, x_{i} I_{i} t \bar{r}$. For each $S \in \mathcal{S}$, let $E_{\bar{r}}\left(R_{N}, S\right)$ be the set of $\bar{r}$-egalitarian-equivalent allocations in $S$ at $R_{N}$.

A social choice correspondence is a set-valued function $\varphi: \mathcal{R}^{n} \times \mathcal{S} \rightarrow \mathcal{S}$ such that $\varphi\left(R_{N}, S\right) \subseteq S$ for all $\left(R_{N}, S\right) \in \mathcal{R}^{n} \times \mathcal{S}$. A social choice correspondence is interpreted as follows: Let $S \in \mathcal{S}$ be the set of allocations available, which may be termed the environment following Arrow (1963, p. 15). Then

\footnotetext{
${ }^{2}$ We might impose a resource constraint on $X$. For example, given a total amount of resources $\Omega \in \mathbf{R}_{+}^{m}$, we might define $X:=\left\{x \in \mathbf{R}_{+}^{m n} \mid \sum_{i=1}^{n} x_{i} \leq \Omega\right\}$. All the results in this paper hold with this definition of $X$. However, by not imposing such a constraint on $X$, we cover situations where the total amounts of resources may change.
} 
$\varphi\left(R_{N}, S\right)$ is the set of socially desirable allocations in the given environment $S$ when the preferences of the agents are $R_{N}$. A fundamental example of a social choice correspondence is the Pareto correspondence, denoted by $P$, which associates with each $\left(R_{N}, S\right) \in \mathcal{R}^{n} \times \mathcal{S}$ the set of all Pareto efficient allocations in $S$ at $R_{N}$. The No-Envy correspondence, the Egalitarian-Equivalence correspondence, and the $\bar{r}$-Egalitarian-Equivalence correspondence, denoted by $F, E$, and $E_{\bar{r}}$, respectively, can be defined analogously.

A remark should follow on the domain of social choice correspondences. The domain consists of the preference domain $\mathcal{R}$ and the alternative domain $\mathcal{S}$. As in many contributions in the literature of social choice theory, we assume that $\mathcal{S}$ is the class of all finite subsets of $X$. Our major interest here is not in investigating what are "optimal" allocations in the set of all technologically feasible allocations. There are many situations in which only a finite number of policy options are at issue at any one time. In such situations, we are rather interested in examining "consistency" of social choices at different times, or under expansions, contractions, or partitions of alternatives available at hand. To that end, our choice of $\mathcal{S}$ would be appropriate.

\section{The Axioms}

This section introduces a variety of desirable properties of social choice correspondences, which we call "axioms". In the rest of the paper, we denote by $\varphi$ a social choice correspondence.

The first axiom is familiar. It means that we should always select from Pareto efficient allocations if there are any.

Pareto Efficiency: For all $\left(R_{N}, S\right) \in \mathcal{R}^{n} \times \mathcal{S}$, if $P\left(R_{N}, S\right) \neq \emptyset$, then $\varphi\left(R_{N}, S\right) \subseteq P\left(R_{N}, S\right)$.

The next three axioms require that only equitable allocations should be chosen whenever there are any.

No-Envy: For all $\left(R_{N}, S\right) \in \mathcal{R}^{n} \times \mathcal{S}$, if $F\left(R_{N}, S\right) \neq \emptyset$, then $\varphi\left(R_{N}, S\right) \subseteq$ $F\left(R_{N}, S\right)$.

Egalitarian-Equivalence: For all $\left(R_{N}, S\right) \in \mathcal{R}^{n} \times \mathcal{S}$, if $E\left(R_{N}, S\right) \neq \emptyset$, then $\varphi\left(R_{N}, S\right) \subseteq E\left(R_{N}, S\right)$. 
$\bar{r}$-Egalitarian-Equivalence: For all $\left(R_{N}, S\right) \in \mathcal{R}^{n} \times \mathcal{S}$, if $E_{\bar{r}}\left(R_{N}, S\right) \neq \emptyset$, then $\varphi\left(R_{N}, S\right) \subseteq E_{\bar{r}}\left(R_{N}, S\right)$.

Even if the efficiency criterion is taken as the first principle for social choice, equity criteria should be used when the efficiency criterion is not at all effective as a guide for selection: either when all the available allocations are efficient or when no available allocation is so. Actually, most economists seem to take this standpoint.

P-Conditional No-Envy ${ }^{3}$ : For all $\left(R_{N}, S\right) \in \mathcal{R}^{n} \times \mathcal{S}$, if (i) $P\left(R_{N}, S\right)=S$ or $P\left(R_{N}, S\right)=\emptyset$, and (ii) $F\left(R_{N}, S\right) \neq \emptyset$, then $\varphi\left(R_{N}, S\right) \subseteq F\left(R_{N}, S\right)$.

The next axiom strengthens P-Conditional No-Envy. It means that if either all the available allocations are efficient or no available allocation is efficient, then all the envy-free allocations should be recommended. In other words, it claims that no other criterion than the efficiency and equity criteria defined explicitly above should be involved as a selection principle.

P-Conditional No-Envy Inclusion: For all $\left(R_{N}, S\right) \in \mathcal{R}^{n} \times \mathcal{S}$, if (i) $P\left(R_{N}, S\right)=S$ or $P\left(R_{N}, S\right)=\emptyset$, and (ii) $F\left(R_{N}, S\right) \neq \emptyset$, then $\varphi\left(R_{N}, S\right)=$ $F\left(R_{N}, S\right)$.

By simply replacing the correspondence $F$ with each of the correspondences $E$ and $E_{\bar{r}}$ in the above definitions, we define P-Conditional EgalitarianEquivalence and P-Conditional $\bar{r}$-Egalitarian-Equivalence, respectively, and their corresponding stronger versions.

Turnig now to the equity-first and efficiency-second principle, we define the counterparts of the above axioms. Let a social choice correspondence $\Psi \in\left\{F, E, E_{\bar{r}}\right\}$ be given. (The correspondence $\Psi$ is one of the three "equity correspondences".) If the equity criterion described by $\Psi$ is accepted as the first selection principle, we may still apply the efficiency criterion when all the allocations available are equitable, or when there is no equitable allocation at all.

$\Psi$-Conditional Pareto Efficiency: For all $\left(R_{N}, S\right) \in \mathcal{R}^{n} \times \mathcal{S}$, if (i) $\Psi\left(R_{N}, S\right)=S$ or $\Psi\left(R_{N}, S\right)=\emptyset$, and (ii) $P\left(R_{N}, S\right) \neq \emptyset$, then $\varphi\left(R_{N}, S\right) \subseteq$ $P\left(R_{N}, S\right)$.

\footnotetext{
${ }^{3}$ The capital letter $\mathbf{P}$ stands for the Pareto correspondence.
} 
As an example, when $\Psi=F$, we write the above axiom F-Conditional Pareto Efficiency. A strengthening of $\Psi$-Conditional Pareto Efficiency is following.

$\Psi$-Conditional Pareto Inclusion: For all $\left(R_{N}, S\right) \in \mathcal{R}^{n} \times \mathcal{S}$, if (i) $\Psi\left(R_{N}, S\right)=S$ or $\Psi\left(R_{N}, S\right)=\emptyset$, and (ii) $P\left(R_{N}, S\right) \neq \emptyset$, then $\varphi\left(R_{N}, S\right)=$ $P\left(R_{N}, S\right)$.

Ever since Arrow (1951), it has been a central issue in social choice theory whether we could construct, by a satisfactory way, social choice correspondences that are rational. Here, rationality means that the choice described by the social choice correspondence from each set of available alternatives could be obtained by maximization of some "well-behaved" social preference relation. The question itself is of much theoretical interest, and moreover it is worth examining because various degrees of rationality conditions are logically related to an important choice-consistency condition called Path Independence.

Let $S$ be the set of available allocations, and $\left\{S_{1}, S_{2}\right\}$ be a partition of $S$. Suppose that we first choose desirable allocations $\varphi\left(S_{i}\right)$ from each $S_{i}(i=1,2)$, and next make the final choice from $\varphi\left(S_{1}\right) \cup \varphi\left(S_{2}\right)$. Then, Path Independence requires that for any partition of $S$, the final choice should be the same as $\varphi(S)$, and hence the choice be independent of the way how to partition $S$. Therefore, path independent social choice rules are immune to any agenda control.

Path Independence: For all $R_{N} \in \mathcal{R}^{n}$, and all $S, S_{1}, S_{2} \in \mathcal{S}$, if $S=S_{1} \cup S_{2}$ and $S_{1} \cap S_{2}=\emptyset$, then $\varphi\left(R_{N}, \varphi\left(R_{N}, S_{1}\right) \cup \varphi\left(R_{N}, S_{2}\right)\right)=\varphi\left(R_{N}, S\right)$.

Path Independence implies the following condition, which was introduced by Chernoff (1954). Its intuitive meaning is also clear: If an allocation is chosen from a bigger set $S_{1}$, and it is still available in a smaller set $S_{2} \subset S_{1}$, then the allocation should be selected from the set $S_{2}$ as well.

Chernoff's Axiom: For all $R_{N} \in \mathcal{R}^{n}$ and all $S_{1}, S_{2} \in \mathcal{S}$, if $S_{2} \subseteq S_{1}$, then $S_{2} \cap \varphi\left(S_{1}\right) \subseteq \varphi\left(S_{2}\right)$.

Next we introduce several rationality conditions of social choice correspondences, and describe their logical relations to Path Independence and Chernoff's Axiom. Let $\succ$ be an irreflexive and asymmetric binary relation 
on $X$, the interpretation of which is a strict social preference relation. ${ }^{4}$ For each $S \in \mathcal{S}$, denote by $M_{\succ}(S)$ the set of maximal elements of $\succ$ in $S$, that is,

$$
M_{\succ}(S):=\{x \in S \mid \text { There exists no } y \in S \text { such that } y \succ x\}
$$

Quasi-Transitive Rationality: ${ }^{5}$ For every $R_{N} \in \mathcal{R}^{n}$, there exists an irreflexive, asymmetric, and transitive binary relation $\succ_{\left(R_{N}\right)}$ on $X$ such that for all $S \in \mathcal{S}, \varphi\left(R_{N}, S\right)=M_{\succ_{\left(R_{N}\right)}}(S)$.

A weaker requirement than Quasi-Transitive Rationality is Acyclic Rationality. Let $\succ$ be an irreflexive and asymmetric binary relation on $X$. We say that $\succ$ has a cycle if there exist an integer $K \geq 3$ and $K$ allocations $x^{1}, \cdots, x^{K}$ such that $x^{k+1} \succ x^{k}$ for all $k$ with $1 \leq k<K$, and $x^{1} \succ x^{K}$. The binary relation $\succ$ is acyclic if it has no cycle.

Acyclic Rationality: For every $R_{N} \in \mathcal{R}^{n}$, there exists an irreflexive, asymmetric, and acyclic binary relation $\succ_{\left(R_{N}\right)}$ on $X$ such that for all $S \in \mathcal{S}$, $\varphi\left(R_{N}, S\right)=M_{\succ\left(R_{N}\right)}(S)$.

It is well-known that given a binary relation $\succ$ on $X$, the set $M_{\succ}(S)$ is non-empty for all finite subsets $S$ of $X$ if and only if $\succ$ is acyclic.

The conditions introduced above have the following logical relations. ${ }^{6}$ Quasi-Transitive Rationality implies both Acyclic Rationality and Path Independence, and each of the two conditions, Acyclic Rationality and Path Independence, implies Chernoff's Axiom. (See Figure 1.) Hence, Chernoff's Axiom may be considered as the minimal requirement on rationality of social choice correspondences.

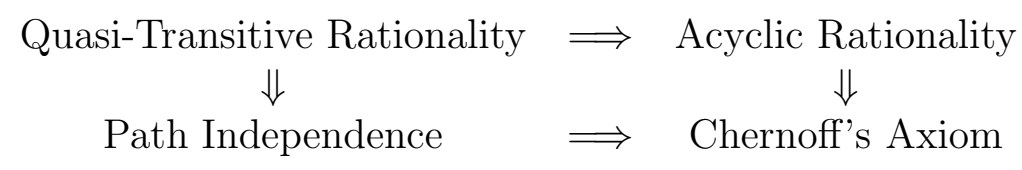

Figure 1: Logical relations of rationality conditions

\footnotetext{
${ }^{4}$ It will be convenient for us to present the results by strict social preference relations $\succ$. However, we could alternatively use the reflexive and complete social preference relations $\succeq$ induced from $\succ$ as follows: For all $x, y \in X, x \succeq y$ if and only if $y \succ x$ does not hold.

"The term "quasi-transitivity" is due to Sen (1970), which means transitivity of strict social preference relations.

${ }^{6}$ See Suzumura (1983, Ch. 3).
} 
Our final axiom is an obvious requirement: Social choice rules should be able to select some allocations for any environment.

Non-Emptiness: For all $R_{N} \in \mathcal{R}^{n}$ and all $S \in \mathcal{S}, \varphi\left(R_{N}, S\right) \neq \emptyset$.

\section{Conflicts between Efficiency and Equity}

This section reviews the fundamental conflict between the Pareto efficiency criterion and the equity criteria. First, we observe the conflict between efficiency and no-envy. Consider the following example, which is essentially due to Feldman and Kirman (1974).

Example 1 There are two agents $N=\{1,2\}$ and two goods $\{1,2\}$. The preferences of the agents are represented by the utility functions

$$
\begin{aligned}
& u_{1}\left(x_{11}, x_{12}\right)=x_{11} x_{12} \\
& u_{2}\left(x_{21}, x_{22}\right)=2 x_{21}+x_{22}
\end{aligned}
$$

Let $x=\left(x_{1}, x_{2}\right)=\left(\left(1, \frac{46}{5}\right),\left(9, \frac{4}{5}\right)\right)$ and $y=\left(y_{1}, y_{2}\right)=\left(\left(\frac{11}{5}, \frac{22}{5}\right),\left(\frac{39}{5}, \frac{28}{5}\right)\right.$. Then, since $u_{1}\left(y_{1}\right)>u_{1}\left(x_{1}\right)$ and $u_{2}\left(y_{2}\right)>u_{2}\left(x_{2}\right)$, the allocation $y$ is Pareto superior to the allocation $x$. However, $x$ is envy-free because $u_{1}\left(x_{1}\right)>u_{1}\left(x_{2}\right)$ and $u_{2}\left(x_{2}\right)>u_{2}\left(x_{1}\right)$, whereas $y$ is not since $u_{1}\left(y_{2}\right)>u_{1}\left(y_{1}\right) \cdot{ }^{7}$ Now let $S=$ $\{x, y\}$. Then, both the sets $P\left(R_{N}, S\right)$ and $F\left(R_{N}, S\right)$ are non-empty, but the intersection of the two sets is empty.

This example can be extended to the case of any finite numbers of agents and goods. Hence, we have the following impossibility.

Theorem 1 [Feldman and Kirman (1974), Suzumura (1981a)] There exists no social choice correspondence that satisfies Non-Emptiness, Pareto Efficiency, and No-Envy.

Next we show that the same kind of conflict may also arise between the Pareto efficiency criterion and the equity-as-egalitarian-equivalence criterion.

\footnotetext{
${ }^{7}$ Moreover, at $y$, agent 2 receives more of every good than agent 1 . Hence, even the nodomination condition, which is a weaker requirement of equity than no-envy, is violated. (An allocation $x \in X$ satisfies no-domination if there exist no $i, j \in N$ such that $x_{i k}>x_{j k}$ for all $k=1, \cdots, m$. Under monotonicity of preference relations, no-envy implies nodomination.)
} 
Example 2 There are two agents $N=\{1,2\}$ and two goods 1,2. The preferences of the agents are represented by the utility functions ${ }^{8}$

$$
\begin{aligned}
& u_{1}\left(x_{11}, x_{12}\right)=\min \left\{x_{11}, x_{12}\right\} \\
& u_{2}\left(x_{21}, x_{22}\right)=x_{21}+3 x_{22}
\end{aligned}
$$

Let $x=\left(x_{1}, x_{2}\right)=((3,11),(9,1))$ and $y=\left(y_{1}, y_{2}\right)=((8,8),(4,4))$. Let $\bar{r}=(1,1)$. Then, the allocation $y$ is Pareto superior to the allocation $x$. On the other hand, since $u_{1}\left(x_{1}\right)=3=u_{1}(3 \bar{r})$ and $u_{2}\left(x_{2}\right)=12=u_{2}(3 \bar{r})$, the allocation $x$ is $\bar{r}$-egalitarian-equivalent with $3 \bar{r}$ being the reference bundle. However, $y$ is not egalitarian-equivalent because for any bundle $z_{0}$ such that $u_{1}\left(z_{0}\right)=u_{1}\left(x_{1}\right), z_{0} \geq(8,8)$ and hence $u_{2}\left(z_{0}\right) \geq u_{2}(8,8)>u_{2}(4,4)=u_{2}\left(y_{2}\right)$.

The above example also shows that the equity-as- $\bar{r}$-egalitarianequivalence criterion may conflict with the Pareto efficiency criterion.

Theorem 2 (i) There exists no social choice correspondence that satisfies Non-Emptiness, Pareto Efficiency, and Egalitarian-Equivalence. (ii) There exists no social choice correspondence that satisfies Non-Emptiness, Pareto Efficiency, and $\bar{r}$-Egalitarian-Equivalence.

\section{The Efficiency-First Principle}

When two criteria of decision-makings are mutually inconsistent, it is natural to take one criterion as the first principle and the other as the second. In this section, we accept the efficiency criterion as the first, keeping the requirement of Pareto Efficiency on social choice correspondences. As for equity criteria, however, we only require their conditional versions: the equity criteria should be applied when the efficiency criterion is not at all effective as a guide for selection, i.e., either when all the allocations available are efficient or when there is no efficient allocation.

By the definitions of axioms, Pareto Efficiency and P-Conditional No-Envy (or P-Conditional Egalitarian-Equivalence, P-Conditional $\bar{r}$ Egalitarian-Equivalence) together are compatible with Non-Emptiness. Then, we examine with which rationality or choice-consistency conditions these axioms are compatible. In order to present the results, we introduce some social preference relations.

\footnotetext{
${ }^{8}$ We use Leontief preferences only for easy calculations. An example can be constructed with smooth and strictly monotonic preferences
} 


\subsection{No-Envy as the Second Criterion}

Let $R_{N} \in \mathcal{R}^{n}$ be given. We say that two allocations $x \in X$ and $y \in X$ are Pareto noncomparable at $R_{N}$ if neither $x$ nor $y$ is Pareto superior to the other. We also say that an allocation $x$ is equity-as-no-envy superior to an allocation $y$ at $R_{N}$ if $x$ is envy-free but $y$ is not. Two allocations $x$ and $y$ are equity-as-no-envy noncomparable at $R_{N}$ if neither $x$ nor $y$ is equity-as-no-envy superior to the other.

Given $R_{N} \in \mathcal{R}^{n}$, define the binary relation $\succ_{P F\left(R_{N}\right)}$ on $X$ as follows: For all $x, y \in X, x \succ_{P F\left(R_{N}\right)} y$ if and only if (i) $x$ is Pareto superior to $y$ at $R_{N}$, or (ii) $x$ and $y$ are Pareto noncomparable and $x$ is equity-as-no-envy superior to $y$ at $R_{N}$. We call $\succ_{P F\left(R_{N}\right)}$ the binary relation of efficiency-first and equity-second-as-no-envy at $R_{N}$.

Under the social preference relation $\succ_{P F\left(R_{N}\right)}$, we first apply the Pareto superior criterion when we rank any two allocations. Then, only when the two allocations are noncomparable in the Pareto superior criterion, we apply the equity-as-no-envy criterion.

The next lemma clarifies the relation between the social preference relation $\succ_{P F\left(R_{N}\right)}$ and the social choice correspondences satisfying Pareto Efficiency, P-Conditional No-Envy, and Chernoff's Axiom.

Lemma 1 If a social choice correspondence $\varphi$ satisfies Pareto Efficiency, $P$ Conditional No-Envy, and Chernoff's Axiom, then $\varphi\left(R_{N}, S\right) \subseteq M_{\succ_{P F\left(R_{N}\right)}}(S)$ for all $\left(R_{N}, S\right) \in \mathcal{R}^{n} \times \mathcal{S}$.

Proof. Suppose that a social choice correspondence $\varphi$ satisfies Pareto Efficiency, P-Conditional No-Envy, and Chernoff's Axiom. Let $\left(R_{N}, S\right) \in \mathcal{R}^{n} \times \mathcal{S}$ be given. Suppose, on the contrary, that there exists $x \in S$ such that $x \in \varphi\left(R_{N}, S\right)$ but $x \notin M_{\succ_{P F\left(R_{N}\right)}}(S)$. Then, there exists $y \in S$ such that $y \succ_{P F\left(R_{N}\right)} x$. Because $x \in \varphi\left(R_{N}, S\right) \subseteq P\left(R_{N}, S\right)$ by Pareto Efficiency, $y$ is not Pareto superior to $x$. Hence, $y \succ_{P F\left(R_{N}\right)} x$ holds only if $x$ and $y$ are Pareto noncomparable, and $y$ is envy-free whereas $x$ is not. Let $S^{\prime}=\{x, y\}$. Then, $S^{\prime} \subseteq S$, and $P\left(R_{N}, S^{\prime}\right)=S^{\prime}$. By P-Conditional NoEnvy, $\varphi\left(R_{N}, S^{\prime}\right) \subseteq F\left(R_{N}, S^{\prime}\right)$. Thus, $x \notin \varphi\left(R_{N}, S^{\prime}\right)$. This means, however, that $\varphi$ violates Chernoff's Axiom, which is a contradiction. Q.E.D.

For any binary relation $\succ$, the set $M_{\succ}(S)$ is non-empty for all $S \in \mathcal{S}$ if and only if $\succ$ is acyclic. Hence, it follows from the above lemma that 
there exist social choice correspondences satisfying the three axioms and NonEmptiness only if $\succ_{P F\left(R_{N}\right)}$ is acyclic. Unfortunately, the social preference relation $\succ_{P F\left(R_{N}\right)}$ may have a cycle as the next proposition shows.

Proposition 1 There exist a preference profile $R_{N} \in \mathcal{R}^{n}$ such that the binary relation of efficiency-first and equity-second-as-no-envy at $R_{N}, \succ_{P F\left(R_{N}\right)}$, has a cycle.

Proof. For simplicity of presentation, we consider a two-agent and two-good economy. Similar examples can be constructed for the case of any numbers of agents and goods. Let $N=\{1,2\}$ be the set of agents. Assume that each agent $i \in N$ has the preference relation $R_{i}$ on $\mathbf{R}_{+}^{2}$ that is represented by the following utility function $u_{i}$ :

$$
\begin{aligned}
& u_{1}\left(x_{11}, x_{12}\right)=x_{11} x_{12} \\
& u_{2}\left(x_{21}, x_{22}\right)=2 x_{21}+x_{22}
\end{aligned}
$$

Define four allocations $x, y, z$ and $w$ by $x=((1,9),(9,1)), y=$ $((3,6),(7,4)), z=((2,8),(8,2))$, and $w=((2,7),(8,3))$. Then, $x \succ_{P F\left(R_{N}\right)} y$ since $x$ and $y$ are Pareto-noncomparable, and $x$ is envy-free but $y$ is not so. Since $y$ is Pareto superior to $z$, we have $y \succ_{P F\left(R_{N}\right)} z$. Because $z$ and $w$ are Pareto-noncomparable, and $z$ is envy-free while $w$ is not, we have $z \succ_{P F\left(R_{N}\right)} w$. Finally, $w \succ_{P F\left(R_{N}\right)} x$ follows from the fact that $w$ is Pareto superior to $x$. Thus, the relation $\succ_{P F\left(R_{N}\right)}$ has a cycle. Q.E.D.

From Lemma 1 and Proposition 1, the next impossibility theorem follows.

Theorem 3 There exists no social choice correspondence that satisfies NonEmptiness, Pareto Efficiency, P-Conditional No-Envy, and Chernoff's Axiom.

We argued that Path Independence is an indispensable property of social choice correspondences. However, since Path Independence implies Chernoff's Axiom, we have the following impossibility as a corollary of Theorem 3 .

Corollary 1 There exists no social choice correspondence that satisfies NonEmptiness, Pareto Efficiency, P-Conditional No-Envy, and Path Independence. 


\subsection{Egalitarian-Equivalence as the Second Criterion}

Next, we adopt egalitarian-equivalence as the concept of equity instead of noenvy. The analyses will go parallel to those in the previous subsection. We define the binary relation of efficiency-first and equity-second-as-egalitarianequivalence at $R_{N} \in \mathcal{R}^{n}$, denoted $\succ_{P E\left(R_{N}\right)}$, by simply replacing in the definition of $\succ_{P F\left(R_{N}\right)}$ the "equity-as-no-envy superior" relation with the "equityas-egalitarian-equivalence superior" relation.

Just as Lemma 1, we can show that if a social choice correspondence $\varphi$ satisfies Pareto Efficiency, P-Conditional Egalitarian-Equivalence, and Chernoff's Axiom, then $\varphi\left(R_{N}, S\right) \subseteq M_{\succ_{P E\left(R_{N}\right)}}(S)$ for all $\left(R_{N}, S\right) \in \mathcal{R}^{n} \times \mathcal{S}$. Hence, whether the social preference relation $\succ_{P E\left(R_{N}\right)}$ is acyclic or not is a crucial question for the existence of social choice correspondence satisfying the three axioms and Non-Emptiness. However, $\succ_{P E\left(R_{N}\right)}$ may have a cycle.

Proposition 2 There exists a preference profile $R_{N} \in \mathcal{R}^{n}$ such that the binary relation of efficiency-first and equity-second-as-egalitarian-equivalence at $R_{N}, \succ_{P E\left(R_{N}\right)}$, has a cycle.

Proof. For simplicity of presentation, we consider a two-agent and two-good economy. Let $N=\{1,2\}$ be the set of agents. Agent 1's preference relation $R_{1}$ on $\mathbf{R}_{+}^{2}$ is represented by a Leontief utility function:

$$
u_{1}\left(x_{11}, x_{12}\right)=\min \left\{x_{11}, x_{12}\right\}
$$

Agent 2's preference relation $R_{2}$ is represented by the following piece-wise linear utility function:

$$
\begin{aligned}
& u_{2}\left(x_{21}, x_{22}\right)=x_{21}+20 \text { if } x_{22} \geq x_{21} \text { and } x_{22} \geq 20 \\
& u_{2}\left(x_{21}, x_{22}\right)=x_{21}+x_{22} \text { if } x_{22} \geq x_{21} \text { and } x_{22} \leq 20 \\
& u_{2}\left(x_{21}, x_{22}\right)=2 x_{22} \text { if } x_{22} \leq x_{21}
\end{aligned}
$$

Define four allocations $x, y, z$ and $w$ by $x=((18,9),(10,19)), y=$ $((12,10),(16,18)), z=((23,11),(5,17))$ and $w=((17,15),(11,13))$. Then, observe the following facts:

(1) The allocation $y$ is Pareto superior to the allocation $x$.

(2) Also, $w$ is Pareto superior to $z$.

(3) However, $y$ and $z$ are Pareto noncomparable.

(4) Similarly, $x$ and $w$ are Pareto noncomparable. 
(5) On the other hand, the allocation $x$ is egalitarian-equivalent with a reference bundle $(9,20)$ since $u_{1}\left(x_{1}\right)=9=u_{1}(9,20)$ and $u_{2}\left(x_{2}\right)=29=u_{2}(9,20)$.

(6) Similarly, $z$ is egalitarian equivalent with a reference bundle $(11,11)$.

(7) However, $y$ is not egalitarian-equivalent because for all $a_{0} \in \mathbf{R}_{+}^{2}$ such that $u_{2}\left(a_{0}\right)=u_{2}\left(y_{2}\right)=34, a_{0} \geq(13,13)$, and hence $u_{1}\left(a_{0}\right) \geq u_{1}(13,13)=13>$ $10=u_{1}\left(y_{1}\right)$.

(8) Similarly, $w$ is not egalitarian-equivalent since for all $b_{0} \in \mathbf{R}_{+}^{2}$ such that $u_{1}\left(b_{0}\right)=u_{1}\left(w_{1}\right)=15, b_{0} \geq(15,15)$, and thus $u_{2}\left(b_{0}\right) \geq u_{2}(15,15)=30>$ $24=u_{2}\left(w_{2}\right)$.

By (1), we have $y \succ_{P E\left(R_{N}\right)} x$. It follows from (3), (6) and (7) that $z \succ_{P E\left(R_{N}\right)} y$. By (2), w $\succ_{P E\left(R_{N}\right)} z$. Finally, from (4), (5) and (8) together, we have $x \succ_{P E\left(R_{N}\right)} w$. Thus, the relation $\succ_{P E\left(R_{N}\right)}$ has a cycle. Q.E.D.

By Proposition 2, we have the following impossibility results.

Theorem 4 There exists no social choice correspondence that satisfies NonEmptiness, Pareto Efficiency, P-Conditional Egalitarian-Equivalence, and Chernoff's Axiom.

Corollary 2 There exists no social choice correspondence that satisfies NonEmptiness, Pareto Efficiency, P-Conditional Egalitarian-Equivalence, and Path Independence.

\section{$5.3 \quad \bar{r}$-Egalitarian-Equivalence as the Second Criterion}

We have reached an impossibility again with egalitarian-equivalence as the second criterion. In this subsection, we adopt a more restricted concept of equity than egalitarian-equivalence, namely $\bar{r}$-egalitarian-equivalence. Recall that the reference bundle of any $\bar{r}$-egalitarian-equivalent allocation must lie in the direction of the given vector $\bar{r}$, while there is no such restriction in the definition of (general) egalitarian-equivalent allocations. With $\bar{r}$-egalitarianequivalence as the second criterion, we have a positive result as shown next.

We define the binary relation of efficiency-first and equity-second-as- $\bar{r}$ egalitarian-equivalence at $R_{N} \in \mathcal{R}^{n}$, denoted $\succ_{P E_{\bar{r}}\left(R_{N}\right)}$, by replacing in the definition of $\succ_{P F\left(R_{N}\right)}$ the "equity-as-no-envy superior" relation with the "equity-as- $\bar{r}$-egalitarian-equivalence superior" relation. As in Lemma 1 , it can be shown that if a social choice correspondence $\varphi$ satisfies Pareto Efficiency, P-Conditional $\bar{r}$-Egalitarian-Equivalence, and Chernoff's Axiom, then $\varphi\left(R_{N}, S\right) \subseteq M_{\succ_{P E_{\bar{r}}\left(R_{N}\right)}}(S)$ for all $\left(R_{N}, S\right) \in \mathcal{R}^{n} \times \mathcal{S}$. 
Proposition 3 For all $R_{N} \in \mathcal{R}^{n}$, the binary relation of efficiency-first and equity-second-as-r-egalitarian-equivalence at $R_{N}, \succ_{P E_{\bar{r}}\left(R_{N}\right)}$, is acyclic.

To prove the above proposition, we first establish the following lemma.

Lemma 2 Let $R_{N} \in \mathcal{R}^{n}$ be given. Suppose that there exist an integer $K>2$ and $K$ allocations $x^{1}, \cdots, x^{K} \in X$ such that for all $k \in\{1, \cdots, K-1\}$, $x^{k+1} \succ_{P E_{\bar{r}}\left(R_{N}\right)} x^{k}$. Suppose further that there exist $k_{1}, k_{2} \in\{1, \cdots, K\}$ with $k_{1}<k_{2}$ such that $x^{k_{1}} \in E_{\bar{r}}\left(R_{N}, X\right), x^{k_{2}} \in E_{\bar{r}}\left(R_{N}, X\right)$, and for all integers $k$ with $k_{1}<k<k_{2}, x^{k} \notin E_{\bar{r}}\left(R_{N}, X\right)$. Then, for all $i \in N, x_{i}^{k_{2}} \succ_{i} x^{k_{1}}$.

Proof. We distinguish two cases.

Case 1: $k_{2}=k_{1}+1$.

Because both of $x^{k_{1}}$ and $x^{k_{2}}$ are $\bar{r}$-egalitarian-equivalent, $x^{k_{2}} \succ_{P E_{\bar{r}}\left(R_{N}\right)} x^{k_{1}}$ holds only if $x^{k_{2}}$ is Pareto superior to $x^{k_{1}}$. Hence, there is an agent $j \in N$ such that (1) $x_{j}^{k_{2}} P_{j} x_{j}^{k_{1}}$. Since $x^{k_{1}}, x^{k_{2}} \in E_{\bar{r}}\left(R_{N}, X\right)$, there are $\lambda_{1}, \lambda_{2} \in \mathbf{R}$ such that $x_{i}^{k_{1}} I_{i} \lambda_{1} \bar{r}$ and $x_{i}^{k_{2}} I_{i} \lambda_{2} \bar{r}$ for all $i \in N$, and in particular for the above agent $j$. Together with (1), this implies that $\lambda_{2}>\lambda_{1}$ by monotonicity of preferences. Therefore, for all $i \in N, x_{i}^{k_{2}} I_{i} \lambda_{2} \bar{r} P_{i} \lambda_{1} \bar{r} I_{i} x_{i}^{k_{1}}$.

Case 2: $k_{2}>k_{1}+1$.

For all integers $k$ with $k_{1}+1 \leq k \leq k_{2}-1, x^{k} \notin E_{\bar{r}}\left(R_{N}, X\right)$. Hence, for all $k$ with $k_{1} \leq k \leq k_{2}-2, x^{k+1} \succ_{P E_{\bar{r}}\left(R_{N}\right)} x^{k}$ holds only if $x^{k+1}$ is Pareto superior to $x^{k}$. By the transitivity of the Pareto superior relation, we have $(2) x^{k_{2}-1}$ is Pareto superior to $x^{k_{1}}$. Since $x^{k_{2}} \succ_{P E_{\bar{r}}\left(R_{N}\right)} x^{k_{2}-1}$ and $x^{k_{2}} \in E_{\bar{r}}\left(R_{N}, X\right)$, either (3) $x^{k_{2}}$ is Pareto superior to $x^{k_{2}-1}$ or (4) $x^{k_{2}}$ and $x^{k_{2}-1}$ are Pareto noncomparable. If (4) is the case and $x_{i}^{k_{2}} I_{i} x_{i}^{k_{2}-1}$ for all $i \in N$, then $x^{k_{2}-1}$ becomes $\bar{r}$-egalitarian-equivalent, which contradicts the supposition. Thus, in either case of (3) and (4), there is at least one agent $j \in N$ such that $x_{j}^{k_{2}} P_{j} x_{j}^{k_{2}-1}$, and together with $(2), x_{j}^{k_{2}} P_{j} x_{j}^{k_{1}}$. The rest of the argument is the same as in Case 1, and we omit it.

Q.E.D.

Proof of Proposition 3. Let $R_{N} \in \mathcal{R}^{n}$ be given. To lighten notation, we simply write $\succ_{P E_{\bar{r}}}, \succ_{P}$, and $E_{\bar{r}}$ for $\succ_{P E_{\bar{r}}\left(R_{N}\right)}, \succ_{P\left(R_{N}\right)}$, and $E_{\bar{r}}\left(R_{N}, X\right)$, respectively. Suppose that there exist an integer $K>2$ and $K$ allocations $x^{1}, \cdots, x^{K}$ such that for all $k \in\{1, \cdots, K-1\}, x^{k+1} \succ_{P E_{\bar{r}}} x^{k}$. We want to show that it is not true that $x^{1} \succ_{P E_{\bar{r}}} x^{K}$.

If $x^{k} \notin E_{\bar{r}}$ for all $k \in\{1, \cdots, K\}$, then $x^{k+1} \succ_{P} x^{k}$ for all $k \in\{1, \cdots, K-$ $1\}$, and by the transitivity of $\succ_{P}$, we have $x^{K} \succ_{P} x^{1}$ and so $x^{K} \succ_{P E_{\bar{r}}} x^{1}$.

Suppose that there exists $k \in\{1, \cdots, K\}$ with $x^{k} \in E_{\bar{r}}$. Let $k^{*}:=$ $\min \left\{k \mid x^{k} \in E_{\bar{r}}\right\}$ and $k^{* *}:=\max \left\{k \mid x^{k} \in E_{\bar{r}}\right\}$. We distinguish two cases. 
Case 1: $k^{*}=1$.

If $k^{* *}=K$, then by using Lemma 2 (repeatedly), we have $x^{K} \succ_{P} x^{1}$, and $x^{K} \succ_{P E_{\bar{r}}} x^{1}$. Suppose that $k^{* *}<K$. Then for all integers $k$ with $k^{* *}+1 \leq$ $k \leq K, x^{k} \notin E_{\bar{r}}$, and hence $x^{k} \succ_{P E_{\bar{r}}} x^{k-1}$ holds only if $x^{k} \succ_{P} x^{k-1}$. Thus, (1) $x^{K} \succ_{P} x^{k^{* *}}$. If $k^{* *}=k^{*}=1$, then $x^{K} \succ_{P} x^{1}$. Otherwise, by Lemma 2, we have that $x^{k^{* *}} \succ_{P} x^{k^{*}}=x^{1}$, and together with (1), $x^{K} \succ_{P} x^{1}$. Hence, $x^{K} \succ_{P E_{\bar{r}}} x^{1}$.

Case 2: $k^{*}>1$.

We prove by way of contradiction that $x^{1} \succ_{P E_{\bar{r}}} x^{K}$ does not hold. Suppose, on the contrary, that (2) $x^{1} \succ_{P E_{\bar{r}}} x^{K}$. Then, since $x^{1} \notin E_{\bar{r}}$, (2) holds only if (3) $x^{1} \succ_{P} x^{K}$. Let $k$ be an integer with $k \neq k^{*}$. (4) If $1<k<k^{*}$ or $k^{* *}<k \leq K$, then $x^{k} \notin E_{\bar{r}}$, and $x^{k} \succ_{P} x^{k-1}$ must hold. (5) By Lemma 2 , if $k^{* *} \neq k^{*}$, then $x^{k^{* *}} \succ_{P} x^{k^{*}}$. By the transitivity of $\succ_{P},(3)$, (4) and (5) together imply that $x^{k^{*}-1} \succ_{P} x^{k^{*}}$. But this means that $x^{k^{*}-1} \succ_{P E_{\bar{r}}} x^{k^{*}}$, which is a contradiction.

Q.E.D.

A positive result follows from the above proposition.

Theorem 5 There exists a social choice correspondence that satisfies NonEmptiness, Pareto Efficiency, P-Conditional $\bar{r}$-Egalitarian-Equivalence, and Acyclic Rationality.

Proof. Define the social choice correspondence $\varphi_{P E_{\bar{r}}}$ by

$$
\varphi_{P E_{\bar{r}}}\left(R_{N}, S\right)=M_{\succ_{P E_{\bar{r}}\left(R_{N}\right)}}(S) \text { for all }\left(R_{N}, S\right) \in \mathcal{R}^{n} \times \mathcal{S}
$$

Let $\left(R_{N}, S\right) \in \mathcal{R}^{n} \times \mathcal{S}$ be given. Since $S$ is a finite set, $P\left(R_{N}, S\right) \neq \emptyset$. For any $x \in S$, if $x \notin P\left(R_{N}, S\right)$, then there exists $y \in S$ that is Pareto superior to $x$, and hence $y \succ_{P E_{\bar{r}}\left(R_{N}\right)} x$, which implies $x \notin M_{\succ_{P E_{\bar{r}}\left(R_{N}\right)}}(S)=\varphi_{P E_{\bar{r}}}\left(R_{N}, S\right)$. Thus, $\varphi_{P E_{\bar{r}}}\left(R_{N}, S\right) \subseteq P\left(R_{N}, S\right)$. Because this relation holds for all $\left(R_{N}, S\right) \in$ $\mathcal{R}^{n} \times \mathcal{S}, \varphi_{P E_{\bar{r}}}$ satisfies Pareto Efficiency. By a similar argument, we can show that $\varphi_{P E_{\bar{r}}}$ satisfies P-Conditional $\bar{r}$-Egalitarian-Equivalence. Finally, Acyclic Rationality and Non-Emptiness follow from Proposition 3 and the definition of $\varphi_{P E_{\bar{r}}}$.

Q.E.D.

It can be checked that the social choice correspondence $\varphi_{P E_{\bar{r}}}$ defined in the above proof satisfies $E_{\bar{r}}$-Conditional Pareto Inclusion as well. However, it violates Path Independence. In fact, here is another impossibility.

Theorem 6 There exists no social choice correspondence that satisfies NonEmptiness, Pareto Efficiency, P-Conditional $\bar{r}$-Egalitarian-Equivalence, $E_{\bar{r}}$ Conditional Pareto Inclusion, and Path Independence. 
Proof. Consider the economy as defined in the proof of Proposition 2. Let $\bar{r}=(9,20)$. Define three allocations $x, y$ and $w$ by $x=((18,9),(10,19)), y=$ $((12,10),(16,18))$ and $w=((17,15),(11,13))$. Then, the allocation $x$ is $\bar{r}$ egalitarian-equivalent, but the other two allocations are not. On the other hand, the allocation $y$ is Pareto superior to the allocation $x$, but $y$ and $w$ are Pareto noncomparable. The allocations $x$ and $w$ are Pareto noncomparable either.

Suppose that there exists a social choice correspondence $\varphi$ that satisfies Pareto Efficiency, P-Conditional $\bar{r}$-Egalitarian-Equivalence, $E_{\bar{r}}$-Conditional Pareto Inclusion, Path Independence, and Non-Emptiness. It follows from Pareto Efficiency, P-Conditional $\bar{r}$-Egalitarian-Equivalence, and Path Independence (which implies Chernoff's Axiom) that

$$
\varphi\left(R_{N}, S\right) \subseteq M_{\succ_{P E_{\bar{r}}\left(R_{N}\right)}}(S) \text { for all }\left(R_{N}, S\right) \in \mathcal{R}^{n} \times \mathcal{S}
$$

Let $S_{1}=\{x, y, w\}, S_{2}=\{x, y\}$ and $S_{3}=\{w\}$. Then, $M_{\succ_{P E_{\bar{r}}\left(R_{N}\right)}}\left(S_{1}\right)=\{y\}$ and $M_{\succ_{P E_{\bar{r}}\left(R_{N}\right)}}\left(S_{2}\right)=\{y\}$. By the relation (1) and Non-Emptiness of $\varphi$, we have

$$
\varphi\left(R_{N}, S_{1}\right)=\{y\} \text { and } \varphi\left(R_{N}, S_{2}\right)=\{y\}
$$

By Non-Emptiness, $\varphi\left(R_{N}, S_{3}\right)=\{w\}$. Hence,

$$
\varphi\left(R_{N}, \varphi\left(R_{N}, S_{2}\right) \cup \varphi\left(R_{N}, S_{3}\right)\right)=\varphi\left(R_{N},\{y, w\}\right)
$$

Observe that $E_{\bar{r}}\left(R_{N},\{y, w\}\right)=\emptyset$ and $P\left(R_{N},\{y, w\}\right)=\{y, w\}$. Since $\varphi$ satisfies $E_{\bar{r}}$-Conditional Pareto Inclusion, we have

$$
\varphi\left(R_{N},\{y, w\}\right)=\{y, w\}
$$

It follows from (2), (3) and (4) that $\varphi\left(R_{N}, \varphi\left(R_{N}, S_{2}\right) \cup \varphi\left(R_{N}, S_{3}\right)\right) \neq$ $\varphi\left(R_{N}, S_{1}\right)$. This means that $\varphi$ violates Path Independence, which is a contradiction.

Q.E.D.

\section{The Equity-First Principle}

In this section, we reverse the order of application of the efficiency and equity criteria. That is, we first apply an equity criterion to select allocations, and then the efficiency criterion only conditionally. The results turn out to be 
remarkably different from those obtained in the previous section. In the following, we only consider the equity-as-no-envy criterion. Essentially the same results hold true with the other two equity concepts.

To identify the social choice correspondences satisfying No-Envy, FConditional Pareto Efficiency and Chernoff's Axiom, a social preference relation is introduced here.

Given $R_{N} \in \mathcal{R}^{n}$, define the binary relation $\succ_{F P\left(R_{N}\right)}$ on $X$ as follows: For all $x, y \in X, x \succ_{F P\left(R_{N}\right)} y$ if and only if (i) $x$ is equity-as-no-envy superior to $y$ at $R_{N}$, or (ii) $x$ and $y$ are equity-as-no-envy noncomparable and $x$ is Pareto superior to $y$ at $R_{N}$. We call $\succ_{F P\left(R_{N}\right)}$ the binary relation of equityfirst-as-no-envy and efficiency-second at $R_{N}$. Under this social preference relation, we first apply the equity-no-envy criterion when we rank any two allocations. Then, only when the two allocations are not comparable in the equity-as-no-envy criterion, the efficiency criterion is used to rank them.

The next lemma is the counterpart of Lemma 1.

Lemma 3 If a social choice correspondence $\varphi$ satisfies No-Envy, FConditional Pareto Efficiency, and Chernoff's Axiom, then $\varphi\left(R_{N}, S\right) \subseteq$ $M_{\succ_{F P\left(R_{N}\right)}}(S)$ for all $\left(R_{N}, S\right) \in \mathcal{R}^{n} \times \mathcal{S}$.

Proof. Suppose that a social choice correspondence $\varphi$ satisfies No-Envy, F-Conditional Pareto Efficiency, and Chernoff's Axiom. Let $\left(R_{N}, S\right) \in \mathcal{R}^{n} \times$ $\mathcal{S}$ be given. Suppose, on the contrary, that there exists $x \in S$ such that $x \in \varphi\left(R_{N}, S\right)$ but $x \notin M_{\succ_{F P\left(R_{N}\right)}}(S)$. Then, there exists $y \in S$ such that $y \succ_{F P\left(R_{N}\right)} x$. We distinguish two cases.

Case 1: $F\left(R_{N}, S\right) \neq \emptyset$.

Because $x \in \varphi\left(R_{N}, S\right) \subseteq F\left(R_{N}, S\right)$ by No-Envy, $y \succ_{F P\left(R_{N}\right)} x$ holds only if both $x$ and $y$ are envy-free, and $y$ is Pareto superior to $x$. Let $S^{\prime}=\{x, y\}$. Then, $S^{\prime} \subseteq S$, and $F\left(R_{N}, S^{\prime}\right)=S^{\prime}$. By F-Conditional Pareto Efficiency, $\varphi\left(R_{N}, S^{\prime}\right) \subseteq P\left(R_{N}, S^{\prime}\right)=\{y\}$. Thus, $x \notin \varphi\left(R_{N}, S^{\prime}\right)$. This means that $\varphi$ violates Chernoff's Axiom, which is a contradiction.

Case 2: $F\left(R_{N}, S\right)=\emptyset$.

Then, $y \succ_{F P\left(R_{N}\right)} x$ holds only if $y$ is Pareto superior to $x$. Let $S^{\prime}=\{x, y\}$. Then, $S^{\prime} \subseteq S$, and $F\left(R_{N}, S^{\prime}\right)=\emptyset$. By F-Conditional Pareto Efficiency, $\varphi\left(R_{N}, S^{\prime}\right) \subseteq P\left(R_{N}, S^{\prime}\right)=\{y\}$. The rest of the argument is the same as Case 1.

Q.E.D.

Proposition 4 For all $R_{N} \in \mathcal{R}^{n}$, the binary relation of equity-first-as-noenvy and efficiency-second at $R_{N}, \succ_{F P\left(R_{N}\right)}$, is transitive. 
Proof. Let $R_{N} \in \mathcal{R}^{n}$ be given. To lighten notation, we simply write $\succ_{F P}$, $\succ_{P}$, and $F$ for $\succ_{F P\left(R_{N}\right)}, \succ_{P\left(R_{N}\right)}$, and $F\left(R_{N}, X\right)$, respectively. Assume that $x \succ_{F P} y$ and $y \succ_{F P} z$. By $x \succ_{F P} y$,

(1) $x \in F$ and $y \notin F$ or

(2) $[[x \in F$ and $y \in F]$ or $[x \notin F$ and $y \notin F]]$ and $x \succ_{P} y$

By $y \succ_{F P} z$,

(3) $y \in F$ and $z \notin F$ or

(4) $[[y \in F$ and $z \in F]$ or $[y \notin F$ and $z \notin F]]$ and $y \succ_{P} z$

(1) and (3) are incompatible. If (1) and (4) hold, then we must have $x \in F$ and $z \notin F$. Hence, $x \succ_{F P} z$. Similarly, if (2) and (3) hold, then $x \in F$ but $z \notin F$, and we have $x \succ_{F P} z$. If (2) and (4) hold, then either $x, y, z \in F$ and $x \succ_{P} y \succ_{P} z$ or $x, y, z \notin F$ and $x \succ_{P} y \succ_{P} z$. Since the relation $\succ_{P}$ is transitive, we have $x \succ_{F P} z$.

Q.E.D.

The next possibility theorem relies on the above proposition.

Theorem 7 There exists a social choice correspondence that satisfies NonEmptiness, No-Envy, F-Conditional Pareto Efficiency, and Quasi-Transitive Rationality.

Proof. Define the social choice correspondence $\varphi_{F P}$ by

$$
\varphi_{F P}\left(R_{N}, S\right)=M_{\succ_{F P\left(R_{N}\right)}}(S) \text { for all }\left(R_{N}, S\right) \in \mathcal{R}^{n} \times \mathcal{S}
$$

Then, by a similar argument to the proof of Theorem 5, we can show that $\varphi_{F P}$ satisfies No-Envy and F-Conditional Pareto Efficiency. By Proposition $4, \varphi_{F P}$ satisfies Quasi-Transitive Rationality. Since any quasi-transitive binary relation has maximal elements in any finite set, Non-Emptiness follows.

Q.E.D.

Since Quasi-Transitive Rationality implies Path Independence, the next corollary follows from Theorem 7 . This corollary should be contrasted with Corollary 1.

Corollary 3 There exists a social choice correspondence that satisfies NonEmptiness, No-Envy, F-Conditional Pareto Efficiency, and Path Independence. 
In the proof of Theorem 7, we defined the social choice correspondence $\varphi_{F P}$ as $\varphi_{F P}\left(R_{N}, S\right)=M_{\succ_{F P\left(R_{N}\right)}}(S)$ for all $\left(R_{N}, S\right) \in \mathcal{R}^{n} \times \mathcal{S}$. This correspondence takes the following values:

$$
\begin{aligned}
& \varphi\left(R_{N}, S\right)=P\left(R_{N}, F\left(R_{N}, S\right)\right) \text { if } F\left(R_{N}, S\right) \neq \emptyset \\
& \varphi\left(R_{N}, S\right)=P\left(R_{N}, S\right) \text { if } F\left(R_{N}, S\right)=\emptyset
\end{aligned}
$$

Note that for all $\left(R_{N}, S\right) \in \mathcal{R}^{n} \times \mathcal{S}, P\left(R_{N}, F\left(R_{N}, S\right)\right) \supseteq P\left(R_{N}, S\right) \cap F\left(R_{N}, S\right)$ and there are $\left(R_{N}, S\right) \in \mathcal{R}^{n} \times \mathcal{S}$ such that $P\left(R_{N}, F\left(R_{N}, S\right)\right) \neq P\left(R_{N}, S\right) \cap$ $F\left(R_{N}, S\right)$. Our final result is a characterization of this social choice correspondence.

Theorem 8 A social choice correspondence $\varphi$ satisfies Non-Emptiness, NoEnvy, P-Conditional No-Envy Inclusion, F-Conditional Pareto Inclusion, and Path Independence if and only if $\varphi=\varphi_{F P}$.

Proof. From the proof of Theorem 7, $\varphi_{F P}$ satisfies Non-Emptiness, NoEnvy, F-Conditional Pareto Efficiency, and Path Independence. As easily checked, it also satisfies P-Conditional No-Envy Inclusion and F-Conditional Pareto Inclusion.

Suppose that there is a social choice correspondence $\varphi$ with $\varphi \neq \varphi_{F P}$ that satisfies the five axioms. Then, there is $\left(R_{N}, S\right) \in \mathcal{R}^{n} \times \mathcal{S}$ such that

$$
\varphi\left(R_{N}, S\right) \neq \varphi_{F P}\left(R_{N}, S\right)
$$

If $F\left(R_{N}, S\right)=\emptyset$, then by F-Conditional Pareto Inclusion, $\varphi\left(R_{N}, S\right)=$ $P\left(R_{N}, S\right)$. On the other hand, it can be checked that $\varphi_{F P}\left(R_{N}, S\right)=$ $M_{\succ_{F P\left(R_{N}\right)}}(S)=P\left(R_{N}, S\right)$. Hence, we have $\varphi\left(R_{N}, S\right)=\varphi_{F P}\left(R_{N}, S\right)$, a contradiction. Thus, $F\left(R_{N}, S\right) \neq \emptyset$. Since F-Conditional Pareto Inclusion implies F-Conditional Pareto Efficiency, and Path Independence implies Chernoff's Axiom, it follows from Lemma 3 that

$$
\varphi\left(R_{N}, S\right) \subseteq M_{\succ_{F P\left(R_{N}\right)}}(S)=\varphi_{F P}\left(R_{N}, S\right)
$$

Because $F\left(R_{N}, S\right) \neq \emptyset$, we have

$$
M_{\succ_{F P\left(R_{N}\right)}}(S)=P\left(R_{N}, F\left(R_{N}, S\right)\right)
$$

It follows from (1), (2) and (3) that there exists $x^{*} \in S$ such that $x^{*} \in$ $P\left(R_{N}, F\left(R_{N}, S\right)\right)$ but $x^{*} \notin \varphi\left(R_{N}, S\right)$. Define $S^{\prime}:=\left\{x^{*}\right\} \cup\{y \in S \mid y \in$ 
$F\left(R_{N}, S\right)$ and $\left.x^{*} \succ_{P\left(R_{N}\right)} y\right\} \cup\left[S \backslash F\left(R_{N}, S\right)\right]$. By Lemma $3, \varphi\left(R_{N}, S^{\prime}\right) \subseteq$ $M_{\succ F P\left(R_{N}\right)}\left(S^{\prime}\right)=\left\{x^{*}\right\}$. By Non-Emptiness, we have $\varphi\left(R_{N}, S^{\prime}\right)=\left\{x^{*}\right\}$. Define $S^{\prime \prime}:=S \backslash S^{\prime}$. Again from Lemma 3, it follows that

$$
\varphi\left(R_{N}, S^{\prime \prime}\right) \subseteq M_{\succ_{F P\left(R_{N}\right)}}\left(S^{\prime \prime}\right)=P\left(R_{N}, F\left(R_{N}, S^{\prime \prime}\right)\right)
$$

Claim: $P\left(R_{N}, F\left(R_{N}, S^{\prime \prime}\right)\right) \subset P\left(R_{N}, F\left(R_{N}, S\right)\right)$.

Let $z \in P\left(R_{N}, F\left(R_{N}, S^{\prime \prime}\right)\right)$. Then, $z \in F\left(R_{N}, S^{\prime \prime}\right) \subset F\left(R_{N}, S\right)$. Suppose that $z \notin P\left(R_{N}, F\left(R_{N}, S\right)\right)$. Then, there exists $w \in P\left(R_{N}, F\left(R_{N}, S\right)\right)$ such that $w \succ_{P\left(R_{N}\right)} z$. If $w=x^{*}$, then $z \in S^{\prime}$ and hence $z \notin S^{\prime \prime}$, which is a contradiction. Thus, $w \neq x^{*}$. But then, $w \in S^{\prime \prime}$ and so $z \notin P\left(R_{N}, F\left(R_{N}, S^{\prime \prime}\right)\right)$, which contradicts $z \in P\left(R_{N}, F\left(R_{N}, S^{\prime \prime}\right)\right)$. Therefore, we must have $z \in P\left(R_{N}, F\left(R_{N}, S\right)\right)$. Thus, the claim has been proved.

It follows from (4) and the above claim that $\varphi\left(R_{N}, S^{\prime \prime}\right) \subseteq$ $P\left(R_{N}, F\left(R_{N}, S\right)\right)$. Hence, $\varphi\left(R_{N}, S^{\prime}\right) \cup \varphi\left(R_{N}, S^{\prime \prime}\right)=\left\{x^{*}\right\} \cup \varphi\left(R_{N}, S^{\prime \prime}\right) \subseteq$ $P\left(R_{N}, F\left(R_{N}, S\right)\right)$. Therefore, $P\left(R_{N}, \varphi\left(R_{N}, S^{\prime}\right) \cup \varphi\left(R_{N}, S^{\prime \prime}\right)\right)=\varphi\left(R_{N}, S^{\prime}\right) \cup$ $\varphi\left(R_{N}, S^{\prime \prime}\right)$. Then, by P-Conditional No-Envy Inclusion, we conclude that $\varphi\left(R_{N}, \varphi\left(R_{N}, S^{\prime}\right) \cup \varphi\left(R_{N}, S^{\prime \prime}\right)\right)=F\left(R_{N}, \varphi\left(R_{N}, S^{\prime}\right) \cup \varphi\left(R_{N}, S^{\prime \prime}\right)\right)=\varphi\left(R_{N}, S^{\prime}\right) \cup$ $\varphi\left(R_{N}, S^{\prime \prime}\right)=\left\{x^{*}\right\} \cup \varphi\left(R_{N}, S^{\prime \prime}\right)$. But since $x^{*} \notin \varphi\left(R_{N}, S\right)=\varphi\left(R_{N}, S^{\prime} \cup S^{\prime \prime}\right)$ and $\varphi$ satisfies Path Independence, we must have $x^{*} \notin \varphi\left(R_{N}, \varphi\left(R_{N}, S^{\prime}\right) \cup\right.$ $\left.\varphi\left(R_{N}, S^{\prime \prime}\right)\right)$. This is a contradiction.

Therefore, there is no social choice correspondence $\varphi$ with $\varphi \neq \varphi_{F P}$ that satisfies the five axioms together in the statement of the theorem. Q.E.D.

\section{Concluding Remarks}

In his two seminal papers, Suzumura (1981a, b) examined possibility of constructing social choice correspondences satisfying the following conditions on efficiency and equity as well as choice-consistency.

Fairness Extension: For all $\left(R_{N}, S\right) \in \mathcal{R}^{n} \times \mathcal{S}, \varphi\left(R_{N}, S\right)=P\left(R_{N}, S\right) \cap$ $F\left(R_{N}, S\right)$ if $P\left(R_{N}, S\right) \cap F\left(R_{N}, S\right) \neq \emptyset$.

Fairness Inclusion: For all $\left(R_{N}, S\right) \in \mathcal{R}^{n} \times \mathcal{S}$, if $P\left(R_{N}, S\right) \cap F\left(R_{N}, S\right)=\emptyset$, $y \in \varphi\left(R_{N}, S\right)$, and $x \in S$ is Pareto superior to $y$ or equity-as-no-envy superior to $y$ at $R_{N}$, then $x \in \varphi\left(R_{N}, S\right)$.

Superset Axiom: For all $R_{N} \in \mathcal{R}^{n}$, and all $S_{1}, S_{2} \in \mathcal{S}$, if $S_{1} \subseteq S_{2}$ and $\varphi\left(R_{N}, S_{2}\right) \subseteq \varphi\left(R_{N}, S_{1}\right)$, then $\varphi\left(R_{N}, S_{2}\right)=\varphi\left(R_{N}, S_{1}\right)$. 
Suzumura (1981a) showed that there exists no social choice correspondence satisfying Fairness Extension, Unrestricted Domain, and Superset Axiom. He also established another impossibility theorem that involves Fairness Inclusion and Chernoff's Axiom as the central requirements. Recently, Denicolò (1997) studied compatibility of Fairness Extension with a version of Arrow's Independence of Irrelevant Alternatives.

The first difference between Suzumura (1981a,b) and this paper lies in the domain of social choice correspondences. While he considered a class of abstract social choice problems, we study economic domains. All our results stand on the standard assumptions on agents' preferences in economics. However, the results in this paper involving no-envy as the equity concept can be adapted into the framework of abstract social choice problems. (Egalitarianequivalence can be defined only on the economic domains.)

Secondly, our results are logically independent of Suzumura's impossibility theorems. There are no logical relations between Fairness Extension and any one or any combination of our axioms concerning efficiency and equity. ${ }^{9}$ On the other hand, combined with the requirement of Non-Emptiness, Fairness Inclusion is incompatible with either of our axioms Pareto Efficiency and No-Envy. To explore the reason, let us reconsider the case of fundamental conflict between the Pareto criterion and the equity-as-no-envy criterion as in Section 4. In Example 1, the allocation $y$ is Pareto superior to the allocation $x$, whereas $x$ is equity-as-no-envy-superior to $y$. Then, if a social choice correspondence $\varphi$ satisfies Non-Emptiness and Fairness Inclusion, then $\varphi(\{x, y\})=\{x, y\}$. That is, any correspondence satisfying this axiom avoids selection in face of the fundamental conflict. To the contrary, the correspondences satisfying our efficiency-first or equity-first axioms does make a selection in the case of the fundamental conflict, depending upon which criterion should be placed first.

This paper started with the simple question: "Which criterion should we take first to select socially desirable allocations, the efficiency criterion or the equity criterion?" We have represented two alternative principles in the forms of axioms. We have then examined rationality (or choice-consistency) of the social choice correspondences satisfying these axioms.

The axioms introduced in this paper reflect alternative value judgements when we are faced with the fundamental conflict between efficiency and equity. Some axioms seem to be easily followed by most economists, and others

\footnotetext{
${ }^{9}$ There is no logical relation between Chernoff's Axiom and Superset Axiom, either.
} 
may be accepted as ethically more desirable by philosophers. The objective of the present paper is not to advocate a particular value judgement as the best, but to show how the choice among alternative value judgements affects the possibility of consistent social choices. Indeed, the degree of rationality of social choice correspondences varies widely depending on which principle the correspondences represent. The lack of rationality may be considered as the "cost" of taking a particular value judgement. Of course, the "cost" should be taken into account when making a choice among various philosophical principles. 


\section{References}

[1] Arrow, K. J. (1951, 1963), Social Choice and Individual Values, 1st ed. 1951, 2nd ed. 1963, New Haven, Yale University Press.

[2] Chernoff, H. (1954), "Rational Selection of Decision Functions," Econometrica, Vol. 22, pp. 422-443.

[3] Denicolò, V. (1997), "No-Envy and Arrow's Conditions," forthcoming in Social Choice and Welfare.

[4] Feldman, A. M. and A. Kirman (1974), "Fairness and Envy," American Economic Review, Vol. 64, pp. 996-1005.

[5] Foley, D. (1967), "Resource Allocation and the Public Sector," Yale Economic Essays, Vol. 7, pp. 45-98.

[6] Kolm, S. (1972), Justice et Equité, Paris, Editions du Centre National de la Recherche Scientifique.

[7] Pazner, E. A. and D. Schmeidler (1978), "Egalitarian Equivalent Allocations: A New Concept of Economic Equity," Quarterly Journal of Economics, Vol. 92, pp. 671-687.

[8] Sen, A. K. (1970), Collective Choice and Social Welfare, San Francisco, Holden-Day.

[9] Suzumura, K. (1981a), "On Pareto-Efficiency and the No-Envy Concept of Equity," Journal of Economic Theory, Vol. 25, pp. 367-379.

[10] Suzumura, K. (1981b), "On the Possibility of "Fair" Collective Choice Rule," International Economic Review, Vol. 22, pp. 351-364.

[11] Suzumura, K. (1983), Rational Choice, Collective Decisions, and Social Welfare, Cambridge U.K., Cambridge University Press.

[12] Tinbergen, J. (1953), "Redelijke inkomensverdeling." 